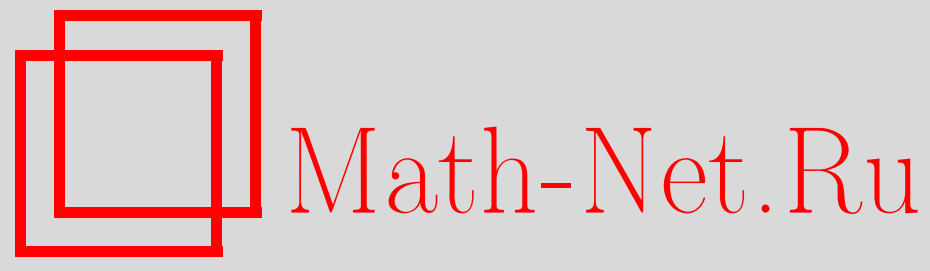

А. Н. Хорин, А. А. Конюхова, Течение Куэтта горячего вязкого газа, Вестн. Сам. гос. техн. ун-та. Сер. Физ.-мат. науки, 2020, номер 2, 365-378

DOI: https://doi.org/10.14498/vsgtu1751

Использование Общероссийского математического портала MathNet.Ru подразумевает, что вы прочитали и согласны с пользовательским соглашением

http://www.mathnet.ru/rus/agreement

Параметры загрузки:

IP : 54.174 .149 .18

26 апреля 2023 г., $15: 32: 20$

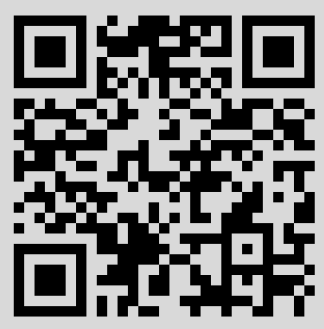


Вестн. Сам. гос. техн. ун-та. Сер. Физ.-мат. науки. 2020. Т. 24, № 2 . С. $365-378$ ISSN: 2310-7081 (online), 1991-8615 (print)

УДК 533.6.011.6, 533.6.011.7, 517.958:531.332

\title{
Течение Куэтта горячего вязкого газа
}

\author{
А. Н. Хорин, А. А. Конюхова \\ Московский физико-технический институт \\ (национальный исследовательский университет), \\ Россия, 141701, Долгопрудный, Институтский пер., 9.
}

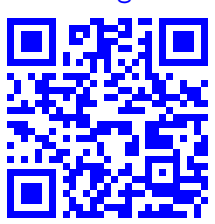

\section{Аннотация}

Найдено новое точное решение уравнений движения вязкого газа для плоского стационарного сдвигового течения горячего (800-1500 K) газа между движущимися с разными скоростями параллельными пластинами (аналог несжимаемого течения Куэтта). Одна из пластин считалась теплоизолированной. Для зависимости коэффициента вязкости от температуры принята формула Сазерленда. В отличие от других известных точных решений, вместо аналогии Рейнольдса (предположение о линейной связи между коэффициентами вязкости и теплопроводности) для вычисления коэффициента теплопроводности использована более точная формула, имеющая в рассматриваемом диапазоне температур ту же точность, что и формула Сазерленда $(2 \%)$. С использованием полученного точного решения исследовано качественное влияние сжимаемости на напряжение трения и на профили температуры и скорости. Показано, что (если одна из пластин теплоизолирована) сжимаемость газа приводит к увеличению напряжения трения. Проведено сравнение нового точного решения с известным точным решением (V. N. Golubkin, G. B. Sizykh, 2018), полученным с использованием формулы Сазерленда для коэффициента вязкости и аналогии Рейнольдса для коэффициента теплопроводности. Обнаружено, что оба решения приводят к одинаковым выводам о качественном влиянии сжимаемости на напряжение трения и на профили температуры и скорости. Однако прирост напряжения трения, вызванный сжимаемостью, при использовании аналогии Рейнольдса оказался недооцененным в два раза. Это показывает, что предположение о линейной связи между коэффициентами вязкости и теплопроводности может приводить к заметным количественным ошибкам.

Ключевые слова: вязкий газ, горячий газ, точные решения, формула Сазерленда, формула для теплопроводности, аналогия Рейнольдса.

Получение: 1 ноября 2019 г. / Исправление: 14 марта 2020 г.

Принятие: 16 марта 2020 г. / Публикация онлайн: 17 июня 2020 г.

\section{Научная статья}

(2) (9) Контент публикуется на условиях лицензии Creative Commons Attribution 4.0 International (https://creativecommons.org/licenses/by/4.0/deed.ru)

\section{Образец для цитирования}

Х о р и н А. Н., К он ю хо в а А. А. Течение Куэтта горячего вязкого газа // Вестн. Сам. гос. техн. ун-та. Сер. Физ.-мат. науки, 2020. Т. 24, № 2. С. 365-378. doi: 10.14498/vsgtu1751.

\section{Сведения об авторах}

Александр Николаевич Хорин (1) https://orcid.org/0000-0002-5251-4930 студент; факультет аэромеханики и летательной техники; e-mail:khorin.an@phystech.edu Анастасия Анатольевна Конюхова (1) https://orcid.org/0000-0002-7872-6974 студент; факультет аэромеханики и летательной техники; e-mail:stasy.mare@gmail.com 
Введение. Одним из первых точных решений, описывающих течения вязкой жидкости, было решение задачи о движении вязкой несжимаемой жидкости между двумя параллельными пластинами, движущимися в своих плоскостях с разными скоростями (течение Куэтта [1]). Уравнения движения вязкого (сжимаемого) газа значительно сложней уравнений Навье-Стокса для вязкой несжимаемой жидкости [2-5]. Поэтому первое точное решение [6] для течения Куэтта вязкого газа удалось получить только при двух упрощающих предположениях. Во-первых, предполагалась линейная связь между коэффициентами вязкости $\mu$ и теплопроводности $\lambda$. Во-вторых, для коэффициента вязкости была принята степенная зависимость от температуры:

$$
\mu=\mu_{0}\left(T / T_{0}\right)^{n}
$$

где $n=0.76$. Позже было получено точное решение [7], в котором оба предположения оставались в силе, но показатель степени $n$ мог быть равен любому числу от 0.5 до 1. Однако более близкой к реальности является не степенная зависимость (1), а формула Сазерленда [2-4]:

$$
\mu=\mu^{*}\left(\frac{T}{T^{*}}\right)^{3 / 2} \frac{T^{*}+T_{S}}{T+T_{S}},
$$

где для воздуха $T^{*}=273 \mathrm{~K}, T_{S}=111 \mathrm{~K}, \mu^{*}=1.715 \cdot 10^{-5} \mathrm{kг} /(\mathrm{м} \cdot \mathrm{c})$. Сравнение с экспериментальными данными, приведенными в таблицах [8], показывает, что точность этой формулы не хуже $2 \%$ для диапазона температур 170-1900 K при давлении менее 10 атм (предполагалось, что воздух сухой и в нем отсутствует диссоциация).

Недавно в статье [9] получено точное решение для течения Куэтта вязкого газа с использованием для вычисления $\mu$ более точной (по сравнению со степенной зависимостью) формулы Сазерленда (2). Однако в [9] осталось в силе предположение о линейной связи между коэффициентами вязкости и теплопроводности. Это предположение считается общепринятым [3,5] и используется до сих пор для различных течений вязкого газа (не только для течения Куэтта) как в теоретических исследованиях [10,11], так и в численных расчетах [12-15]. Однако в недавней статье [16] на основе экспериментальных данных [8] было показано, что отношение $\lambda / \mu$ для воздуха не постоянно и меняется на $3.5 \%$ только в диапазоне $275 \mathrm{~K} \leqslant T \leqslant 375 \mathrm{~K}$, а вне этого диапазона отношение $\lambda / \mu$ меняется еще больше. Следовательно, использование линейной связи коэффициентов $\lambda$ и $\mu$ приводит к ошибке вычисления $\lambda$, превосходящей ошибку вычисления $\mu$ по формуле Сазерленда (2\%). Таким образом, в решении [9] точность вычисления $\lambda$ была хуже точности вычисления $\mu$. В данной статье предпринята попытка улучшить результат [9] путем использования для вычисления $\lambda$ более точной формулы, ошибка которой не превышает $2 \%$.

В настоящее время известны две такие формулы. Одна формула имеет точность $2 \%$ для температур $T<1000 \mathrm{~K}$. Она предложена в монографии [4] и имеет вид

$$
\lambda=\lambda^{*}\left(\frac{T}{T^{*}}\right)^{3 / 2} \frac{T^{*}+T_{\lambda}}{T+T_{\lambda}},
$$

где для воздуха $T^{*}=273 \mathrm{~K}, T_{\lambda}=194 \mathrm{~K}, \lambda^{*}=2.412 \cdot 10^{-2} \mathrm{Bт} /(\mathrm{M} \cdot \mathrm{K})$. Другая 
формула

$$
\lambda=\beta \frac{T-T_{S}}{\sqrt{T}},
$$

где для сухого воздуха $\beta=0.2415 \cdot 10^{-2} \mathrm{~B}_{\mathrm{T}} /\left(\mathrm{M} \cdot \mathrm{K}^{3 / 2}\right)$, предложена в [16] и имеет точность $2 \%$ для горячего $(800 \mathrm{~K} \leqslant T \leqslant 1500 \mathrm{~K})$ вязкого газа.

Использование (3) привело авторов данной статьи к дифференциальному уравнению, точное решение которого получить не удалось. Точное решение, которое приведено ниже, удалось получить с помощью формулы (4).

Таким образом, данная статья посвящена получению точного решения для течения Куэтта вязкого газа с использованием формул (2) и (4), имеющих точность $2 \%$ для горячего $(800 \mathrm{~K} \leqslant T \leqslant 1500 \mathrm{~K})$ газа. Новизна исследования состоит в использовании для вычисления $\lambda$ более точной формулы (4) вместо предположения о линейной связи $\lambda$ и $\mu$.

1. Постановка задачи. Рассмотрим стационарное плоское сдвиговое течение совершенного вязкого газа между двумя движущимися в своих плоскостях параллельными пластинами, расположенными горизонтально на расстоянии $H$ друг от друга (аналог несжимаемого течения Куэтта). Введем прямоугольную декартову систему координат $O x y$, в которой ось $O x$ направлена вдоль нижней пластины, а ось $O y$ перпендикулярна пластинам. Будем считать, что нижняя пластина неподвижна, а верхняя пластина перемещается со скоростью $u_{1}$ (рис. 1). Обозначим: $u$-скорость одномерного течения вдоль оси $x, \rho$ - плотность, $T$ - температура, $\mu=\mu(T), \lambda=\lambda(T)$ - коэффициенты динамической вязкости и теплопроводности газа, определяемые формулами (2) и (4) соответственно.

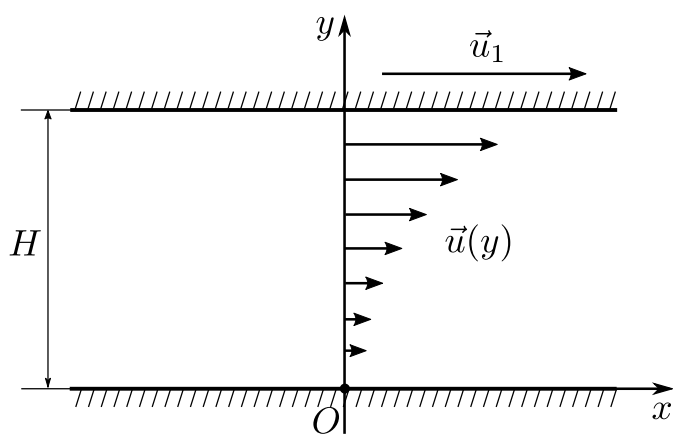

Рис. 1. Сдвиговое течение газа между неподвижной (нижней) и движущейся (верхней) пластинами

[Figure 1. Shear gas flow between the stationary (bottom) and moving (top) plates]

Течение вязкого газа описывается уравнением неразрывности, уравнением импульсов и уравнением баланса энергии [3,4]. Давление, плотность и температура совершенного газа связаны уравнением состояния Менделеева-Клапейрона. Будем считать, что движение газа вызвано только силами вязкости за счет перемещения верхней пластины, а продольный градиент давления отсутствует, т. е. давление постоянно $p=p_{0}=$ const. B pacсматриваемой одномерной задаче скорость, плотность, температура и давление зависят только от координаты $y$. Поэтому уравнение неразрывности выполняется тождественно. Остальные уравнения движения газа приводят к следующей системе: 


$$
\begin{gathered}
\left(\mu u_{y}^{\prime}\right)_{y}^{\prime}=0, \\
\left(\lambda T_{y}^{\prime}\right)_{y}^{\prime}+\mu\left(u_{y}^{\prime}\right)^{2}=0, \\
p=R \rho T=p_{0}>0 .
\end{gathered}
$$

Здесь и далее нижними индексами «0» и «1» обозначаются значения величин на поверхности нижней $(y=0)$ и верхней $(y=H)$ пластин соответственно. $R$ - отношение универсальной газовой постоянной к молярной массе.

Задача состоит в нахождении решения $u, \rho$ и $T$ системы $(2),(4)-(7)$ при заданных значениях $u_{1}, \rho_{0}$ и $T_{0}$.

Как сказано во введении, эта задача отличается от задачи, решенной в [9], формулой для коэффициента теплопроводности. В [9] использована линейная зависимость: $\lambda(T)=\mu(T) \lambda_{0} / \mu_{0}$, а в данной работе- формула (4).

2. Точное решение. Из (5) следует, что напряжение трения $\tau=\mu u_{y}^{\prime}$ одинаково во всех точках течения, то есть $\mu u_{y}^{\prime}=\tau_{0}$. Возьмем в качестве характерных значений плотности, вязкости, теплопроводности и температуры их значения при $y=0$, в качестве характерного значения скорости - скорость верхней пластины $u_{1}$, а в качестве характерной длины - расстояние между пластинами $H$. Перейдем к безразмерным переменным: $\bar{\rho}=\rho / \rho_{0}, \bar{\mu}=\mu / \mu_{0}$, $\bar{\lambda}=\lambda / \lambda_{0}, \bar{T}=T / T_{0}, \bar{T}_{S}=T_{S} / T_{0}, \bar{u}=u / u_{1}, \bar{y}=y / H$, где $\mu_{0}$ и $\lambda_{0}$ вычислены по формулам (2) и (4) для $T=T_{0}$. Тогда для безразмерных коэффициентов вязкости и теплопроводности имеем

$$
\bar{\mu}=\bar{T}^{3 / 2} \frac{1+\bar{T}_{S}}{\bar{T}+\bar{T}_{S}} \quad \text { и } \quad \bar{\lambda}=\frac{\bar{T}-\bar{T}_{S}}{\left(1-\bar{T}_{S}\right) \sqrt{\bar{T}}} .
$$

Введем вместо координаты $\bar{y}$ новую безразмерную переменную $\eta$, выполняя преобразование

$$
\eta=\int_{0}^{\bar{y}} \frac{d \xi}{\bar{T}(\xi)}
$$

предложенное в [16]. В рассматриваемом случае (постоянное давление) это преобразование совпадает с преобразованием Дородницына [5]. Тогда

$$
\frac{d}{d \bar{y}}=\frac{1}{\bar{T}} \frac{d}{d \eta}
$$

и уравнения (5), (6) примут вид (далее всюду штрих будет обозначать дифференцирование по новой безразмерной координате $\eta$ )

$$
\begin{gathered}
\bar{u}^{\prime}=\frac{\tau_{0} H}{u_{1} \mu_{0}} \frac{\bar{T}}{\bar{\mu}} \\
\left(\frac{\bar{\lambda}}{\bar{T}} \bar{T}^{\prime}\right)^{\prime}+\frac{\mu_{0} u_{1}^{2}}{\lambda_{0} T_{0}} \frac{\bar{\mu}}{\bar{T}}\left(u^{\prime}\right)^{2}=0 .
\end{gathered}
$$

С помощью (9) исключим $\bar{u}^{\prime}$ из последнего уравнения:

$$
\left(\frac{\bar{\lambda}}{\bar{T}} \bar{T}^{\prime}\right)^{\prime}+\frac{\tau_{0}^{2} H^{2}}{\mu_{0} \lambda_{0} T_{0}} \frac{\bar{T}}{\bar{\mu}}=0 .
$$


Подстановка выражений (8) в формулу (10) дает

$$
\left(\frac{\bar{T}-\bar{T}_{S}}{\sqrt{\bar{T}}} \frac{1}{\bar{T}} \bar{T}^{\prime}\right)^{\prime}+2 \frac{\bar{T}+\bar{T}_{S}}{\sqrt{\bar{T}}} B^{2}=0
$$

где $B=\tau_{0} H / \sqrt{2\left(1+\bar{T}_{S}\right) \mu_{0} \beta T_{0} \sqrt{T_{0}}}$. Уравнение $(11)$ нелинейно относительно $\bar{T}=\bar{T}(\eta)$. Однако замена

$$
f(\eta)=\frac{\bar{T}(\eta)+\bar{T}_{S}}{\sqrt{\bar{T}(\eta)}}
$$

сводит его к уравнению колебаний

$$
f^{\prime \prime}+B^{2} f=0
$$

Поэтому функция $f(\eta)$ представляется в виде $f(\eta)=A \cos (B \eta+\varphi)$, где $A>0$ и $\varphi \in(-\pi, \pi]$ - некоторые константы, которые будут определены ниже. Из условия $\bar{T}(0)=1$ и из уравнения (12) вытекает связь констант $A$ и $\varphi$ :

$$
A \cos \varphi=1+\bar{T}_{S}
$$

Искомая зависимость $\bar{T}(\eta)$ выражается через функцию $f(\eta)$ из уравнения (12), которое имеет два решения:

$$
\sqrt{\bar{T}(\eta)}=0.5\left(f(\eta) \pm \sqrt{f^{2}(\eta)-4 \bar{T}_{S}}\right) .
$$

Однако условию $\bar{T}(0)=1$ с учетом (13) удовлетворяет только одно из них (при $\left.T_{0}>T_{S}=111 \mathrm{~K}\right)$ :

$$
\begin{aligned}
\sqrt{\bar{T}(\eta)}=0.5(f(\eta)+ & \left.\sqrt{f^{2}(\eta)-4 \bar{T}_{S}}\right)= \\
& =0.5\left(A \cos (B \eta+\varphi)+\sqrt{A^{2} \cos ^{2}(B \eta+\varphi)-4 \bar{T}_{S}}\right)
\end{aligned}
$$

т.e.

$$
\bar{T}(\eta)=0.25 A^{2}\left(\cos (B \eta+\varphi)+\sqrt{\cos ^{2}(B \eta+\varphi)-4 \bar{T}_{S} / A^{2}}\right)^{2}
$$

Из уравнения состояния (7)-

$$
\bar{\rho}(\eta)=1 / \bar{T}(\eta)
$$

При заданном распределении температуры $\bar{T}(\eta)$ плотность определяется по формуле (7), а координата $\bar{y}$ - как интеграл с переменным верхним пределом:

$$
\bar{y}(\eta)=\int_{0}^{\eta} \bar{T}(\xi) d \xi=Y(\eta)-Y(0),
$$

где для точного решения существенно, что этот интеграл берется и первообразная $Y$ имеет явную форму: 


$$
\begin{aligned}
& Y(\eta)=0.25 A^{2} B^{-1}\left(\cos (B \eta+\varphi)+\sqrt{\cos ^{2}(B \eta+\varphi)-\alpha^{2}}\right) \sin (B \eta+\varphi)+ \\
& \quad+0.25 A^{2} B^{-1}\left(1-\alpha^{2}\right)\left((B \eta+\varphi)+\arcsin \left(\frac{\sin (B \eta+\varphi)}{\sqrt{1-\alpha^{2}}}\right)\right), \quad \alpha^{2}=4 \bar{T}_{S} / A^{2} .
\end{aligned}
$$

Поскольку рассматривается только такой диапазон изменения координаты $\eta$, в котором температура $T>0$, формула (16) всегда задает взаимно однозначное соответствие координат $\eta$ и $\bar{y}$.

Из (9) следует, что скорость $\bar{u}$ определяется как интеграл с переменным верхним пределом:

$$
\bar{u}(\eta)=\frac{\tau_{0} H}{u_{1} \mu_{0}} \int_{0}^{\eta} \frac{\bar{T}(\xi)}{\bar{\mu}(\xi)} d \xi .
$$

Подынтегральное выражение, согласно (10), имеет представление

$$
\frac{\bar{T}}{\bar{\mu}}=-\frac{\mu_{0} \lambda_{0} T_{0}}{\tau_{0}^{2} H^{2}}\left(\frac{\bar{\lambda}}{\bar{T}} \bar{T}^{\prime}\right)^{\prime}
$$

Поэтому

$$
\bar{u}(\eta)=-\frac{\lambda_{0} T_{0}}{u_{1} \tau_{0} H} \int_{0}^{\eta}\left(\frac{\bar{\lambda}}{\bar{T}} \bar{T}^{\prime}\right)^{\prime} d \xi=-\left.\frac{\lambda_{0} T_{0}}{u_{1} \tau_{0} H}\left(\frac{\bar{\lambda}}{\bar{T}} \bar{T}^{\prime}\right)\right|_{0} ^{\eta} .
$$

Далее рассмотрим случай теплоизолированной верхней (подвижной) пластины. Это значит, что $\bar{T}^{\prime}\left(\eta_{1}\right)=0$, где $\eta_{1}-$ значение координаты $\eta$ на верхней пластине. Дифференцируя (15), получим

$$
\bar{T}^{\prime}(\eta)=-\frac{2 B \bar{T}(\eta)}{\sqrt{\cos ^{2}(B \eta+\varphi)-\alpha^{2}}} \sin (B \eta+\varphi) .
$$

Поэтому равенство $\bar{T}^{\prime}\left(\eta_{1}\right)=0$ означает, что

$$
\sin \left(B \eta_{1}+\varphi\right)=0
$$

На верхней пластине $\bar{u}\left(\eta_{1}\right)=1$, и уравнение (17) для теплоизолированной верхней пластины после применения (18) дает

$$
1=-\left.\frac{\lambda_{0} T_{0}}{u_{1} \tau_{0} H}\left(\frac{\bar{\lambda}}{\bar{T}} \bar{T}^{\prime}\right)\right|_{0} ^{\eta_{1}}=0+\frac{\lambda_{0} T_{0}}{u_{1} \tau_{0} H} \frac{\bar{\lambda}(0)}{\bar{T}(0)} \bar{T}^{\prime}(0)=-\frac{\lambda_{0} T_{0}}{u_{1} \tau_{0} H} \frac{2 B}{\sqrt{\cos ^{2} \varphi-\alpha^{2}}} \sin \varphi .
$$

Поскольку из (13) следует

$$
\begin{array}{r}
\sqrt{\cos ^{2} \varphi-\alpha^{2}}=\sqrt{\cos ^{2} \varphi-4 \bar{T}_{S} / A^{2}}=\cos \varphi \sqrt{1-4 \bar{T}_{S} /\left(1+\bar{T}_{S}\right)^{2}}= \\
=\cos \varphi\left(1-\bar{T}_{S}\right) /\left(1+\bar{T}_{S}\right)
\end{array}
$$

имеем

$$
1=-\frac{\lambda_{0} T_{0}}{u_{1} \tau_{0} H} \frac{2 B\left(1+\bar{T}_{S}\right) \sin \varphi}{\left(1-\bar{T}_{S}\right) \cos \varphi},
$$

что позволяет явно выразить $\varphi$ через параметры задачи: 


$$
\begin{aligned}
& \operatorname{tg} \varphi=-\frac{u_{1}}{2 \lambda_{0} T_{0}} \frac{\left(1-\bar{T}_{S}\right)}{\left(1+\bar{T}_{S}\right)}\left(\frac{\tau_{0} H}{B}\right)= \\
&=-\frac{u_{1}}{2 \lambda_{0} T_{0}} \frac{\left(1-\bar{T}_{S}\right)}{\left(1+\bar{T}_{S}\right)} \sqrt{2\left(1+\bar{T}_{S}\right) \mu_{0} \beta T_{0} \sqrt{T_{0}}}= \\
&=-\frac{u_{1}\left(1-\bar{T}_{S}\right)}{\lambda_{0} \sqrt[4]{T_{0}}} \sqrt{\frac{\mu_{0} \beta}{2\left(1+\bar{T}_{S}\right)}} .
\end{aligned}
$$

После этого константы $A>0$ и $\alpha^{2}=4 \bar{T}_{S} / A^{2}$ определяются формулой (13).

Для окончательного решения задачи осталось определить константу $B$. Поскольку $\sqrt{\bar{T}(\eta)}>0$ и $A>0$, из (14) заключаем, что $\cos (B \eta+\varphi)>|\alpha|$ при всех $\eta \in\left[0, \eta_{1}\right]$. В частности, при $\eta=0$ имеем $\cos \varphi>|\alpha|$. Вместе с условием $\varphi \in(-\pi, \pi]$ это означает, что $\varphi \in(-\pi / 2, \pi / 2]$, и, следовательно, $(B \eta+\varphi) \in$ $(-\pi / 2, \pi / 2]$ при всех $\eta \in\left[0, \eta_{1}\right]$. Поэтому из (19) следует, что

$$
B \eta_{1}+\varphi=0 .
$$

Учитывая $(20)$ и $(13)$, из (16) и из условия $\bar{y}\left(\eta_{1}\right)=1$ получим

$$
\begin{gathered}
1=Y\left(\eta_{1}\right)-Y(0)=0-Y(0)= \\
=-0.25 A^{2} B^{-1}\left(\cos \varphi+\sqrt{\cos ^{2} \varphi-\alpha^{2}}\right) \sin \varphi- \\
\quad-0.25 A^{2} B^{-1}\left(1-\alpha^{2}\right)\left(\varphi+\arcsin \left(\frac{\sin \varphi}{\sqrt{1-\alpha^{2}}}\right)\right)= \\
=-0.5 A B^{-1} \sin \varphi-0.25 A^{2} B^{-1}\left(1-\alpha^{2}\right)\left(\varphi+\arcsin \left(\frac{\sin \varphi}{\sqrt{1-\alpha^{2}}}\right)\right) .
\end{gathered}
$$

Отсюда получается явное выражение для константы $B$ через другие константы, найденные ранее:

$$
B=-0.5 A \sin \varphi-0.25 A^{2}\left(1-\alpha^{2}\right)\left(\varphi+\arcsin \left(\frac{\sin \varphi}{\sqrt{1-\alpha^{2}}}\right)\right) .
$$

Таким образом, приходим к следующему результату. Для любых положительных значений скорости верхней пластины $u_{1}$, расстояния между пластинами $H$, значений вязкости $\mu_{0}$ и теплопроводности $\lambda_{0}$ на нижней пластине, относительной температуры Сазерленда $\bar{T}_{S}$ и значений температуры нижней пластины $T_{0}$ из заданного диапазона $800 \mathrm{~K} \leqslant T \leqslant 1500 \mathrm{~K}$ формулы (15) и (17) дают точное решение уравнений движения вязкого сжимаемого газа в зависимости от переменной $\eta$. Переход к исходной переменной $\bar{y}$ осуществляется по формуле (16). Это решение описывает вязкое сжимаемое течение Куэтта между нижней неподвижной пластиной и параллельной ей верхней пластиной, движущейся со скоростью $u_{1}$, для общего случая, когда коэффициент вязкости и температура связаны формулой Сазерленда (2), коэффициент теплопроводности и температура связаны формулой (4), а верхняя подвижная пластина теплоизолирована.

В связи со сложностью полученных формул для демонстрации свойств решения в следующих разделах будут приведены профили безразмерных скорости, температуры и напряжения трения $u, T$ и $\tau$. 
3. Профили скорости и температуры. На рис. 2 представлена функция зависимости безразмерного значения температуры $\bar{T}$ от безразмерного значения поперечной координаты $\bar{y}$ при различных значениях параметров $T_{0}$ и $u_{1}$. Как можно видеть из графика, при увеличении скорости верхней пластины $u_{1}$ температура верхней (теплоизолированной) пластины $\left.\bar{T}\right|_{\bar{y}=1}$ возрастает. Однако при постоянном значении $u_{1}$ чем выше значение температуры нижней пластины $T_{0}$, тем незначительнее отличается от нее температура верхней пластины $\left.\bar{T}\right|_{\bar{y}=1}$.

При различных значениях параметров $T_{0}$ и $u_{1}$ профиль скорости близок к прямолинейному отрезку, соединяющему начальную и конечную точки профиля, так что визуально на графике это незаметно. При значительном приближении к центральной части профиля (рис. 3) можно заметить, что по мере увеличения скорости верхней стенки $u_{1}$ и уменьшения температуры нижней стенки $T_{0}$ растет отличие профиля скорости от отрезка прямой.

4. О применимости аналогии Рейнольдса. Как было упомянуто выше, в статье [9] получено точное решение для течения Куэтта вязкого газа с использованием формулы Сазерленда (2). Однако в [9] осталось в силе предположение о линейной связи между коэффициентами вязкости и теплопроводности (аналогия Рейнольдса). Полученное в данной статье точное решение вместе с решением [9] позволило оценить влияние упрощающего предположения о линейной связи коэффициентов вязкости и теплопроводности на результаты расчетов для течения Куэтта. Для этого расчеты профилей температур и зависимостей напряжения трения от числа Маха $\mathrm{M}_{1}$ при теплоизолированной верхней пластине были проведены как по полученным в данной статье формулам, так и по формулам [9].

Сравнение профилей температур проведено при $u_{1}=150$ м/с. Как можно видеть из рис. 4, все выводы, сделанные в предыдущем разделе относительно профилей температуры, справедливы и в случае линейной зависимости. Однако для одинаковых значений температуры нижней пластины $T_{0}$ температура верхней пластины $\left.\bar{T}\right|_{\bar{y}=1}$ в предположении о линейной связи оказывается больше.

5. Напряжение трения. Как показывает уравнение (5), напряжение трения $\tau=\mu u_{y}^{\prime}$ одинаково во всех точках течения и может быть вычислено, например, на нижней пластине. Для исследования влияния сжимаемости введем безразмерный коэффициент напряжения трения $\bar{\tau}_{0}=\tau_{0} / \tau_{00}$, где $\tau_{00}=\left(\mu_{0} u_{1}\right) / H-$ коэффициент напряжения трения для несжимаемого течения Куэтта с постоянным коэффициентом динамической вязкости $\mu_{0}$. А также введем безразмерный коэффициент - число Маха: $\mathrm{M}_{1}=u_{1} / a_{0}$, где $u_{1}$ скорость верхней пластины, а $a_{0}$ - скорость звука на нижней пластине, которая вычисляется по формуле $a_{0}=\sqrt{k R T_{0}}, k=C_{p} / C_{v}$ (для рассматриваемого диапазона температур принималось значение $k=1.365)$. На рис. 5 представлены зависимости безразмерного коэффициента напряжения трения $\bar{\tau}_{0}$ от числа Маха $\mathrm{M}_{1}$, которые вычислены при разных значениях температуры нижней пластины $T_{0}$. Из графиков на рис. 5 следует, что при теплоизолированной верхней пластине сжимаемость газа приводит только к увеличению напряжения трения. Однако при линейной зависимости между коэффициентами вязкости и теплопроводности прирост напряжения трения оказывается недооцененным примерно в два раза.

Заключение. Найдено точное решение уравнений движения горячего 


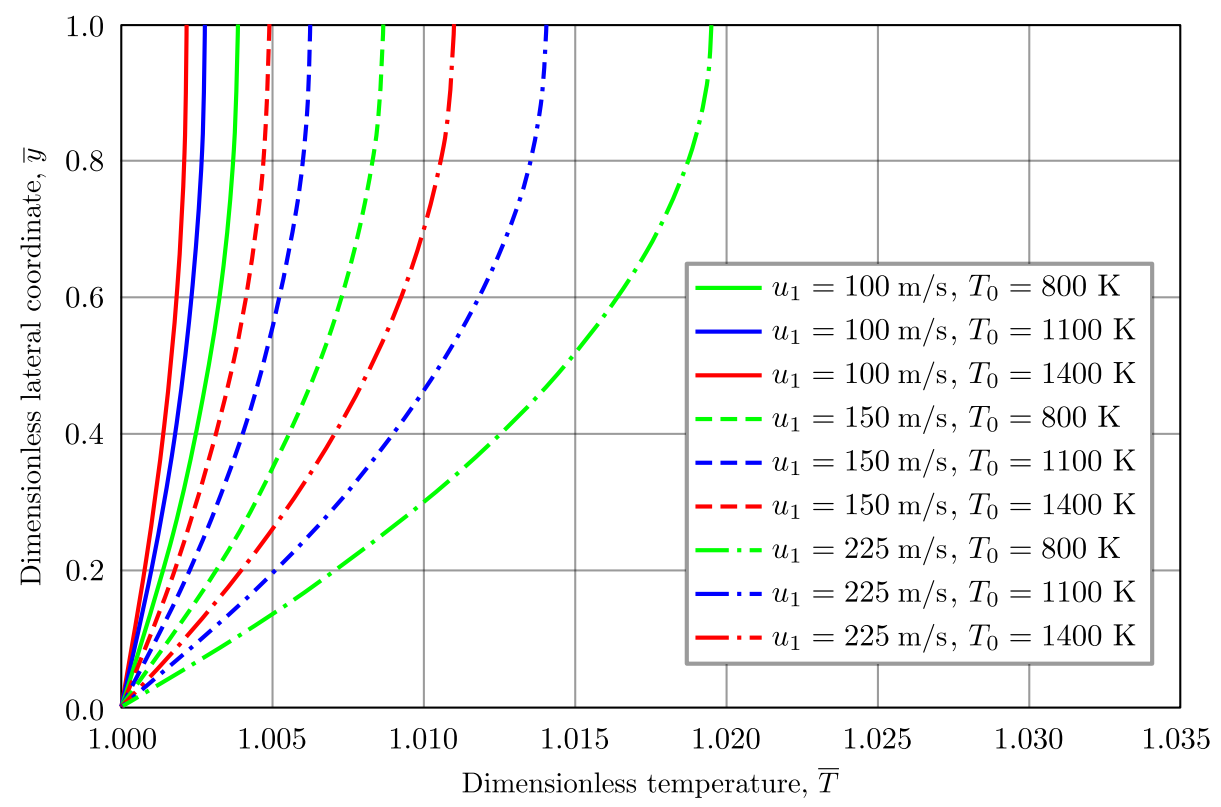

Рис. 2. Профили температур для различных значений $T_{0}$ и $u_{1}$ [Figure 2. The temperature profiles for various values of $T_{0}$ and $u_{1}$ ]

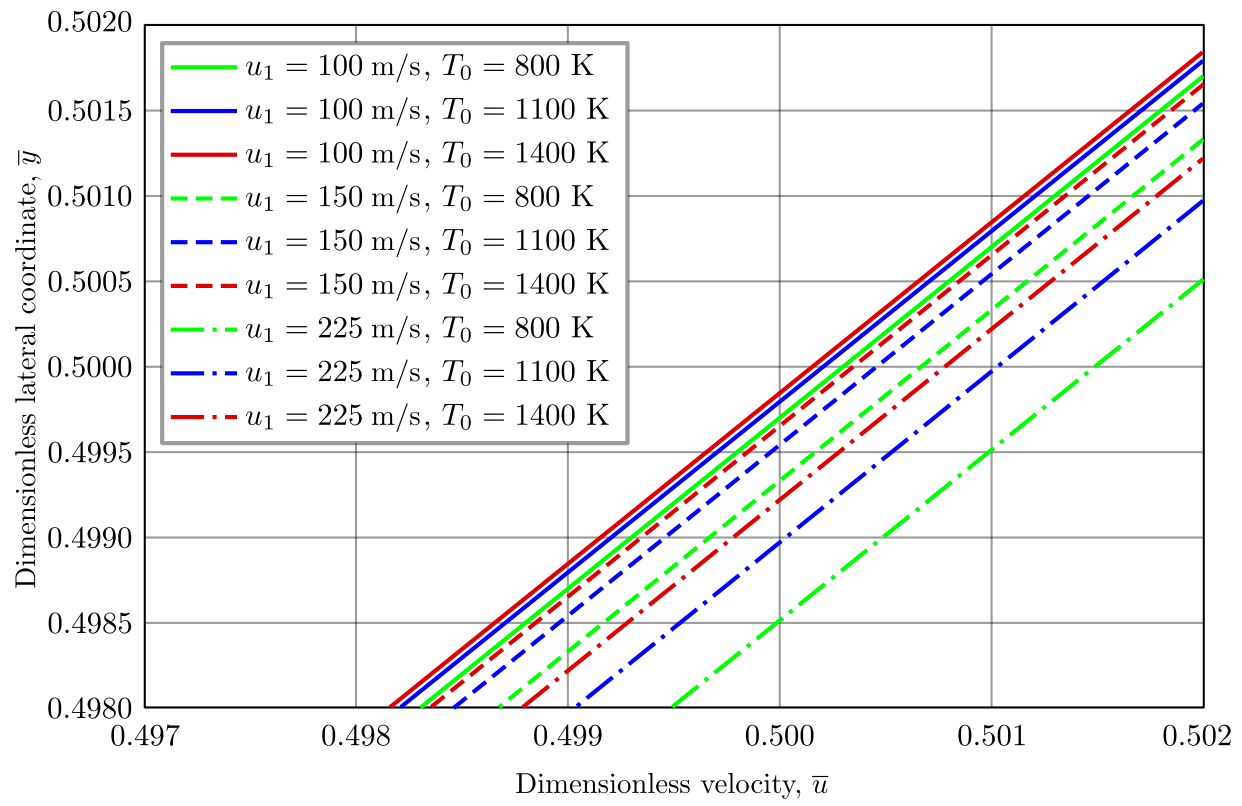

Рис. 3. Центральная часть профилей скорости при значительном приближении для различных значений $T_{0}$ и $u_{1}$

[Figure 3. Central part of velocity profiles with a significant increase for various values of $T_{0}$ and $u_{1}$ ] 


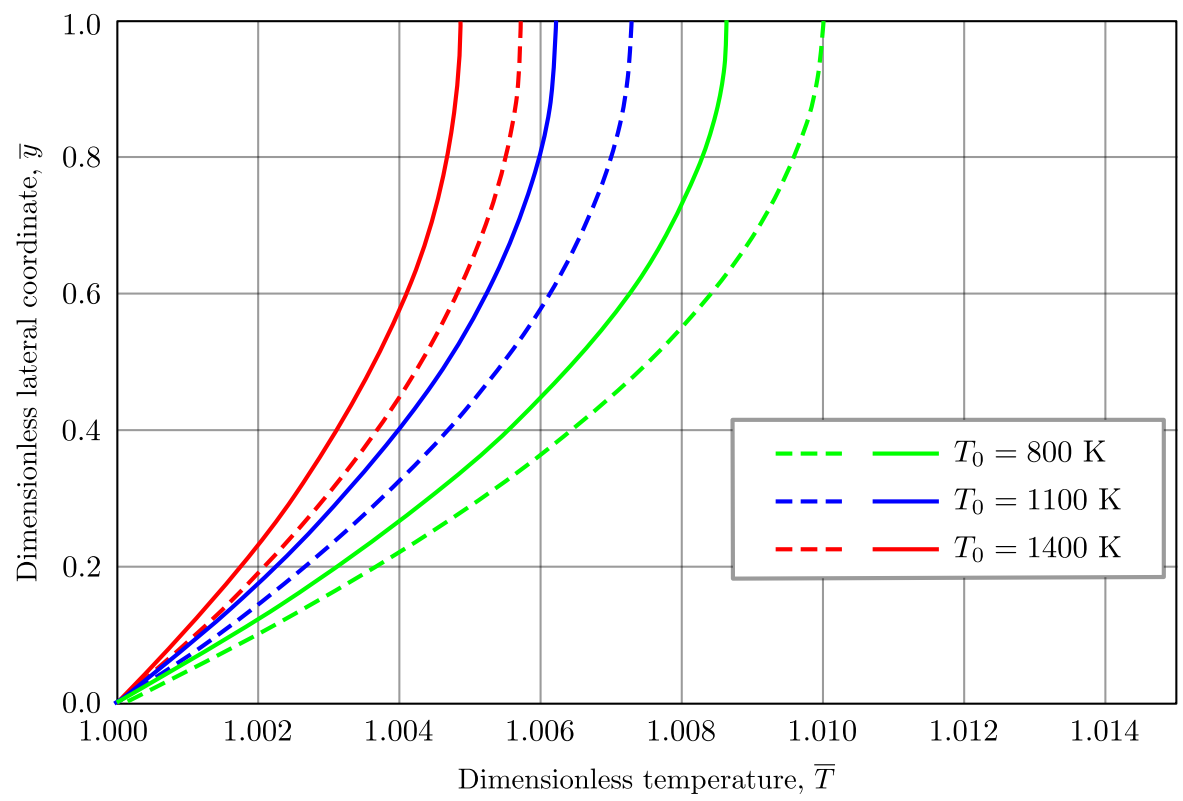

Рис. 4. Профили температур при теплоизолированной верхней пластине для различных значений $T_{0}$ и $u_{1}=150 \mathrm{M} / \mathrm{c} ;$ сплошные линии - по теории авторов; штриховые линии - по данным работы [9]

[Figure 4. The temperature profiles in the presence of thermal insulation on the top plate for various values of $T_{0}$ and $u_{1}=150 \mathrm{~m} / \mathrm{s}$; solid lines are consistent with this study; dashed lines correspond to data from [9]]

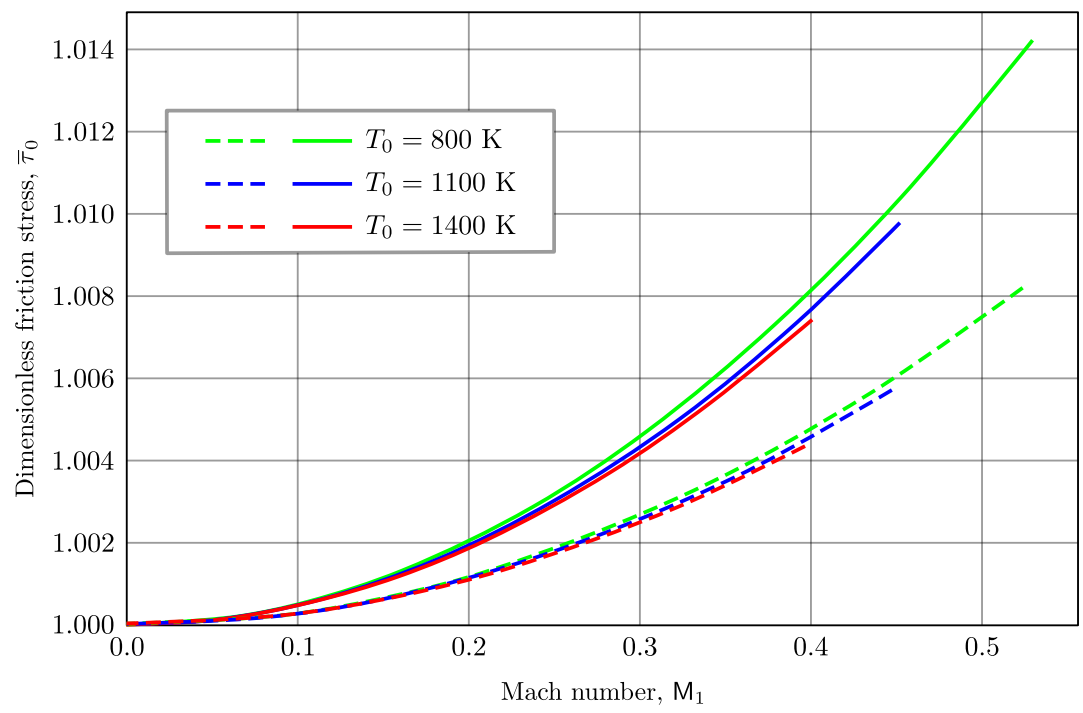

Рис. 5. Зависимость напряжения трения от числа Маха $M_{1}$ при теплоизолированной верхней пластине для различных значений $T_{0}$; сплошные линии - по теории авторов; штриховые линии - по данным работы [9]

[Figure 5. The dependence of the friction stress on the Mach number $M_{1}$ in the presence of thermal insulation on the top plate for various values of $T_{0}$; solid lines are consistent with this study; dashed lines correspond to data from [9]] 
вязкого газа, в которых формулы для вязкости и теплопроводности имеют точность не хуже $2 \%$. Вязкость зависит от температуры по формуле Сазерленда (2), а теплопроводность - по формуле (4). Решение описывает плоское стационарное сдвиговое течение горячего (800 K $\leqslant T \leqslant 1500 \mathrm{~K})$ газа между двумя параллельными пластинами, вызванное движением одной из них (сжимаемое течение Куэтта). Полученное точное решение позволило исследовать качественное влияние сжимаемости на напряжение трения и на профили температуры и скорости. Несмотря на то, что расчеты, проведенные с использованием аналогии Рейнольдса, показывают аналогичные качественные эффекты, в полной мере количественное влияние сжимаемости для течения Куэтта исследовано именно в данной работе.

Конкурирующие интересы. Конкурирующих интересов не имеем.

Авторский вклад и ответственность. Вклад авторов: А. Хорин-60\%, А. Конюхова - $40 \%$. Авторы несут полную ответственность за предоставление окончательной рукописи в печать. Окончательная версия рукописи была одобрена всеми авторами.

Финансирование. Исследование выполнялось без финансирования.

Благодарность. Авторы благодарны рецензентам за тщательное прочтение статьи и ценные предложения и комментарии.

\section{Библиографический список}

1. Couette M. Études sur le frottement des liquides // Ann. Chim. Phys., Ser. 6, 1890. vol. 21. pp. $433-510$.

2. Schlichting H., Gersten K. Grenzschicht-Theorie. Berlin: Springer-Verlag, 2006. doi: 10 . 1007/3-540-32985-4.

3. Лойцянский Л. Г. Механика жидкости и газа. М.-Л.: Гостехиздат, 1950.

4. White F. Viscous Fluid Flow/ Mcgraw-Hill Series in Mechanical Engineering Book Series. New York: McGraw Hill, 2006.

5. Кочин Н. Е., Кибель И. А., Розе Н. В. Теоретическая гидромеханика. Ч. ІІ.. М.: Физматлит, 1963.

6. Гродзовский Г. Л. Течение вязкого газа между двумя движущимися параллельными стенками и между двумя вращающимися цилиндрами // ПММ, 1955. Т. 19, № 1. С. 99102.

7. Жмулин Е. М. Течение вязкого газа между двумя движущимися параллельными пластинами // Уч. записки ЦАГИ, 1971. Т. 2, № 4. С. 31-37.

8. Rogers G. F. C., Mayhew Y. R. Thermodynamic and Transport Properties of Fluids: S.I. Units. Malden, USA: Blackwell, 1995.

9. Голубкин В. Н., Сизых Г. Б. О сжимаемом течении Куэтта // Уч. записки ЦАГИ, 2018. T. 49, № 1. C. 27-38.

10. Брутян М. А., Крапивский П. Л. Точные решения стационарных уравнений НавьеСтокса вязкого теплопроводного газа для плоской струи из линейного источника // ПММ, 2018. Т. 82, № 5. С. 644-656. doi: 10.31857/S003282350002270-7.

11. Брутян М. А., Ибрагимов У. Г. Автомодельные и неавтомодельные течения вязкого газа, истекающего из вершины конуса // Труды МФТИ, 2018. Т. 10, № 4. С. 113-121.

12. Bosnyakov S., Mikhaylov S. V., Morozov A. N., et al. Implementation of high-order discontinuous Galerkin method for solution of practical tasks in external aerodynamics and aeroacoustics / N. Kroll, C. Hirsch, F. Bassi, C. Johnston, K. Hillewaert (eds.), IDIHOM: Industrialization of High-Order Methods - A Top-Down Approach/ Notes on Numerical Fluid Mechanics and Multidisciplinary Design, 128. Cham: Springer, 2015. pp. 337-379. doi : 10.1007/978-3-319-12886-3_16. 
13. Bosnyakov S., Mikhaylov S. V., Podaruev V. Yu., et al. Application of high-order discontinuous Galerkin method to LES/DES test cases using computers with high number of cores / 23rd AIAA Computational Fluid Dynamics Conference (AIAA Aviation 2017, USA, Denver, Colorado, 5-9 June 2017), 2017. pp. 2017-3943. doi: 10.2514/6.2017-3943.

14. Егоров И. В., Новиков А. В. Прямое численное моделирование ламинарнотурбулентного обтекания плоской пластины при гиперзвуковых скоростях потока // Ж. вычисл. матем. и матем. физ., 2016. Т. 56, №6. С. 1064-1081. doi: 10.7868/ S0044466916060120.

15. Егоров И. В., Пальчековская Н. В., Шведченко В. В. Влияние пространственных возмущений сверхзвукового потока на тепловой поток к поверхности затупленных тел // TBT, 2015. T. 53, № 5. C. 713-726. doi: 10.7868/S0040364415040092.

16. Голубкин В. Н., Сизых Г. Б. Течение вязкого газа между вертикальными стенками // ПMM, 2018. Т. 82, № 5. C. 657-667. doi : 10.31857/S003282350002271-8. 


\title{
MSC: 76N15, 80A20
}

\section{Couette flow of hot viscous gas}

\section{A. N. Khorin, A. A. Konyukhova}

\author{
Moscow Institute of Physics and Technology \\ (National Research University), \\ 9, Institutskiy per., Dolgoprudny, 141701, Russian Federation.
}

\begin{abstract}
A new exact solution is found for the equations of motion of a viscous gas for a stationary shear flow of hot (800-1500 K) gas between two parallel plates moving at different speeds (an analog of the incompressible Couette flow). One of the plates was considered thermally insulated. For the dependence of the coefficient of viscosity on temperature, the Sutherland formula is adopted. Unlike other known exact solutions, instead of a linear association between the viscosity and thermal conductivity coefficients, a more accurate formula was used to calculate the thermal conductivity coefficient, having the same accuracy in the temperature range under consideration as the Sutherland formula (2\%). Using the obtained exact solution, the qualitative effect of compressibility on the friction stress and the temperature, and velocity profiles were investigated. It is shown that the compressibility of the gas leads to an increase in the friction stress, if one of the plates is thermally insulated. The new exact solution was compared with the known exact solution (Golubkin, V.N. \& Sizykh, G.B., 2018) obtained using the Sutherland formula for the viscosity coefficient and the Reynolds analogy for the thermal conductivity coefficient. It was found that both solutions lead to the same conclusions about the qualitative effect of compressibility on the friction stress and on the temperature and velocity profiles. However, the increase in friction stress caused by compressibility of the gas turned out to be underestimated twice when using the Reynolds analogy. This shows that the assumption of a linear relationship between the coefficients of viscosity and thermal conductivity can lead to noticeable quantitative errors.
\end{abstract}

Keywords: viscous gas, hot gas, exact solutions, Sutherland formula, thermal conductivity formula.

Received: $1^{\text {st }}$ November, 2019 / Revised: $14^{\text {th }}$ March, $2020 /$ Accepted: $16^{\text {th }}$ March, $2020 /$ First online: $17^{\text {th }}$ June, 2020

\section{Research Article}

() (1) The content is published under the terms of the Creative Commons Attribution 4.0 International License (http://creativecommons.org/licenses/by/4.0/)

\section{Please cite this article in press as:}

Khorin A. N., Konyukhova A. A. Couette flow of hot viscous gas, Vestn. Samar. Gos. Tekhn. Univ., Ser. Fiz.-Mat. Nauki [J. Samara State Tech. Univ., Ser. Phys. Math. Sci.], 2020, vol. 24, no. 2, pp. 365-378. doi: 10.14498/vsgtu1751 (In Russian).

\section{Authors' Details:}

Aleksandr N. Khorin (10) https://orcid.org/0000-0002-5251-4930

Student; Dept. of Aeromechanics and Flight Engineering; e-mail:khorin.an@phystech.edu

Anastasia A. Konyukhova (D) https://orcid.org/0000-0002-7872-6974

Student; Dept. of Aeromechanics and Flight Engineering; e-mail: stasy.mare@gmail.com 
Competing interests. We have no competing interests.

Authors' contributions and responsibilities. Wrote the paper: A. Khorin (60\%) and A. Konyukhova (40\%). The authors are absolutely responsible for submitting the final manuscript in print. Each author has approved the final version of manuscript.

Funding. The research has not had any funding.

Acknowledgments. The authors are grateful to the reviewers for careful reading of the paper and valuable suggestions and comments.

\section{References}

1. Couette M. Études sur le frottement des liquides, Ann. Chim. Phys., Ser. 6, 1890, vol.21, pp. $433-510$.

2. Schlichting H., Gersten K. Grenzschicht-Theorie. Berlin, Springer-Verlag, 2006. doi: 10. 1007/3-540-32985-4.

3. Loitsyanskii L. G. Mechanics of Liquids and Gases. Oxford, Pergamon Press, 1966. doi: 10. 1016/c2013-0-05328-5.

4. White F. Viscous Fluid Flow, Mcgraw-Hill Series in Mechanical Engineering Book Series. New York, McGraw Hill, 2006.

5. Kochin N. E., Kibel I. A., Roze N. V. Theoretical Hydromechanics. New York, Wiley Interscience, 1965.

6. Grodzovskii G. L. Viscous gas flow between two moving parallel walls and between two rotating cylinders, Prikl. Mat. Mekh., 1955, vol.19, no. 1, pp. 99-102 (In Russian).

7. Zhmulin E. M. Viscous gas flow between two moving parallel plates, Uch. Zap. TsAGI, 1971, vol. 2, no. 4, pp. 31-37 (In Russian).

8. Rogers G. F. C., Mayhew Y. R. Thermodynamic and Transport Properties of Fluids: S.I. Units. Malden, USA, Blackwell, 1995.

9. Golubkin V. N., Sizykh G. B. On the compressible Couette flow, TsAGI Science Journal, 2018, vol. 49, no. 1, pp. 29-41. doi: 10.1615/TsAGISciJ. 2018026781.

10. Brutyan M. A., Krapivsky P. L. Exact solutions to the steady Navier-Stokes equations of viscous heat-conducting gas flow induced by the plane jet issuing from the line source, Fluid Dyn., 2018, vol. 53 (Suppl. 2), pp. 1-10. doi : 10.1134/S0015462818060022.

11. Brutyan M. A., Ibragimov U. G. Selfsimilar and nonselfsimilar solutions of the viscous compressible flow inside a cone, Proceedings of MIPT, 2018, vol.10, no. 4, pp. 113-121 (In Russian).

12. Bosnyakov S., Mikhaylov S. V., Morozov A. N., et al. Implementation of high-order discontinuous Galerkin method for solution of practical tasks in external aerodynamics and aeroacoustics, In: N. Kroll, C. Hirsch, F. Bassi, C. Johnston, K. Hillewaert (eds.), IDIHOM: Industrialization of High-Order Methods - A Top-Down Approach, Notes on Numerical Fluid Mechanics and Multidisciplinary Design, 128. Cham, Springer, 2015, pp. 337-379. doi: 10.1007/978-3-319-12886-3_16.

13. Bosnyakov S., Mikhaylov S. V., Podaruev V. Yu., et al. Application of high-order discontinuous Galerkin method to LES/DES test cases using computers with high number of cores, In: 23rd AIAA Computational Fluid Dynamics Conference (AIAA Aviation 2017, USA, Denver, Colorado, 5-9 June 2017), 2017, pp. 2017-3943. doi: 10.2514/6.2017-3943.

14. Egorov I. V., Novikov A. V. Direct numerical simulation of laminar-turbulent flow over a flat plate at hypersonic flow speeds, Comput. Math. Math. Phys., 2016, vol.56, no.6, pp. 1048-1064. doi : 10.1134/S0965542516060129.

15. Egorov I. V., Pal'chekovskaya N. V., Shvedchenko V. V. The effect of spatial perturbations of a supersonic flow on heat flux to the surface of blunt bodies, High Temp., 2015, vol. 53, no. 5, pp. 677-689. doi: 10.1134/S0018151X15040094.

16. Golubkin V. N., Sizykh G. B. Viscous gas flow between vertical walls, Fluid Dyn., 2018, vol. 53 (Suppl. 2), pp. 11-18. doi: 10.1134/S0015462818060046. 